\title{
Constructing Virtual Patient Cohorts for Simulating Atrial Fibrillation Ablation
}

\author{
Caroline H Roney ${ }^{1}$, Marianne Beach ${ }^{1}$, Arihant Mehta ${ }^{1}$, Iain Sim ${ }^{1}$, Cesare Corrado ${ }^{1}$, Rokas Bendikas ${ }^{1}$, \\ Jose A Solis-Lemus ${ }^{1}$, Orod Razeghi ${ }^{1}$, John Whitaker ${ }^{1}$, Louisa O’Neill ${ }^{1}$, Gernot Plank ${ }^{2}$, Edward \\ Vigmond $^{3}$, Steven E Williams ${ }^{1}$, Mark D O'Neill ${ }^{1}$, Steven A Niederer ${ }^{1}$
}

\author{
${ }^{1}$ King's College London, UK. ${ }^{2}$ Medical University of Graz, Austria. ${ }^{3}$ University of Bordeaux, France
}

\begin{abstract}
Determining the optimal treatment approach for patients with atrial fibrillation $(A F)$ is challenging as patient-specific mechanisms underlying the arrhythmia are typically unknown. Virtual patient cohort simulations can be used to investigate these mechanisms and the effects of atrial anatomy, electrical and structural substrate on potential AF ablation treatment outcomes. It is important that virtual cohort models are constructed using a consistent and reproducible approach regardless of the large variability in atrial morphology between patients. This allows comparison of virtual ablation outcomes between cases. In this study we developed a standardised pipeline for constructing personalised biophysical left atrial models using segmented late-gadolinium enhancement magnetic resonance imaging (LGE-MRI) data. Fibrotic remodelling was incorporated according to the distribution of LGE intensity values as changes in conductivity and ionic cell model properties. We present a methodology for simulating AF: seeding four spiral wave re-entries at standard locations across the anatomies; and for testing different ablation approaches across a large virtual patient cohort of personalised left atrial models. We simulated pulmonary vein isolation (PVI) ablation across a cohort of 20 paroxysmal and 30 persistent AF patient models.
\end{abstract}

\section{Introduction}

Atrial fibrillation (AF) is one of the most common cardiac arrhythmias and one potential treatment is catheter ablation therapy. Catheter ablation therapy can be carried out using a variety of approaches, including pulmonary vein isolation (PVI). Whilst in some cases PVI alone is an effective and optimal treatment, it is ineffective in others. Star AF II demonstrated that PVI has a success rate of $59 \%$ at 18 months $(n=67)$. The remaining $41 \%$ of cases may benefit from additional lesions but identifying these patients and their optimal lesion set remains challenging. [1]. Computational modelling could help patient-specific prediction and stratification of $\mathrm{AF}$ patients, leading to improved patient treatment. However, this requires a standardised, rapid and robust modelling pipeline.

Our study developed a modelling pipeline for the construction of personalised anatomical models for simulating atrial fibrillation ablation. We demonstrated the capabilities of the platform by constructing 50 patientspecific biophysical models of AF patients. Finally, we simulated the effects of PVI on AF for each of the models.

\section{Methods}

\subsection{Patient Cohort}

Our study included 20 paroxysmal AF and 30 persistent AF patients. Contrast enhanced magnetic resonance angiogram (CE-MRA) data obtained during patient magnetic resonance imaging (MRI) was used to produce computational models of each patient's left atrial chamber. Further details regarding image acquisition have been published previously [2]. Ethical approval was reviewed and approved by regional ethics committee (17/LO/0150 and $15 / \mathrm{LO} / 1803)$.

\subsection{Personalised anatomical model construction}

We developed a modelling pipeline for the construction of personalised anatomical models. The steps involved in this pipeline are further detailed in the following subsections and represented by Figure 1. This figure shows an example of the model construction, AF simulation and ablation processes involved in the modelling pipeline. The images included are an example of one of the anatomical models of the virtual patient cohort. Grey boxes represent the construction process, the red boxes represent $\mathrm{AF}$ simulation and PS density map generation, and the green boxes demonstrate the post-ablation steps of the modelling pipeline. 


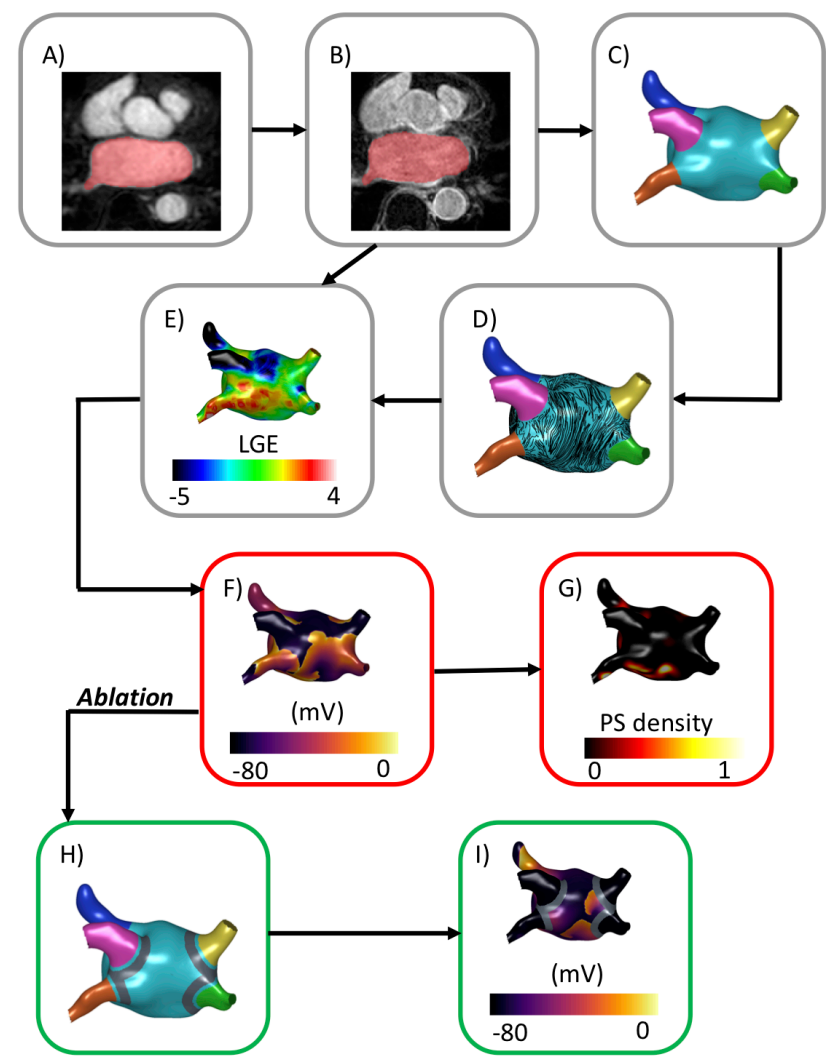

Figure 1. Modelling pipeline for personalised anatomical model construction (A) Anatomical models for each patient were segmented from CE-MRA images. (B) Segmentations were registered with LGE-MRI images. (C) Atrial regions were labelled. (D) Human atrial ex-vivo DTMRI atlas endocardial and epicardial fibres were then incorporated using the Universal Atrial Coordinate system. (E) Fibrotic tissue was added depending on LGE-MRI image intensities. (F) AF was then simulated in the models using Cardiac Arrhythmia Research Package (CARP) software and (G) PS density maps generated. (H) Models were ablated using a PVI ablation approach (grey regions were ablated). (I) Transmembrane potential map $1.5 \mathrm{~s}$ postablation.

\subsubsection{Geometry construction}

Semi-automated tools within CEMRGApp software (www.cemrgapp.com, [2]) were used to segment CE-MRA data and build a left atrial anatomical model for each patient. The segmentation meshes were post-processed using MeshLab and ParaView software. This involved the clipping and labelling of the mitral valve, four pulmonary veins and left atrial appendage [3]. The meshes were then re-meshed to a regular edge length of $0.34 \mathrm{~mm}$ using mmgtools software (www.mmgtools.org). An epicardial surface mesh was produced through duplication and projection of the endocardial surface mesh and linear elements were used to couple these meshes and produce a bilayer model [4]. The Universal Atrial Coordinate system allowed incorporation of a human ex-vivo DTMRI atlas endocardial and epicardial fibre distribution [5] [6]. This is shown in Figure 2.

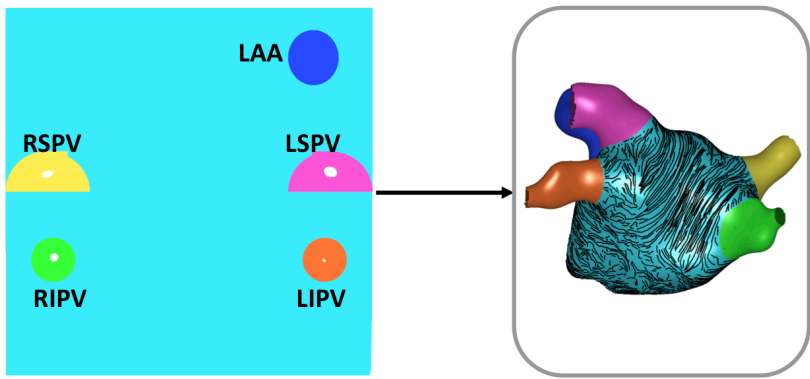

Figure 2. Incorporation of atrial surface fibre fields. Endocardial and epicardial human ex-vivo DTMRI atlas fibres were mapped to the anatomical meshes using the Universal Atrial Coordinate system (left image). This was according to the labels (LAA - left atrial appendage, LSPV - left superior pulmonary vein, LIPV - left inferior pulmonary vein, RSPV - right superior pulmonary vein and RIPV - right inferior pulmonary vein) placed during the processing stages of the model construction process. The LA body endocardial fibre field is shown in the right image.

\subsubsection{Modelling fibrosis}

Fibrotic remodelling was incorporated into the anatomical models through registration of late gadolinium enhancement-magnetic resonance imaging (LGE-MRI) with CE-MRA data. The LGE-MRI data informed the distribution of fibrotic tissue. Fibrotic tissue was modelled through modifications of tissue conductivities together with electrophysiological changes. Conduction velocity (CV) was set to $100 \%$ in regions of $0-56 \%$ normalised LGE intensity; $80 \%$ for $56-60 \%$ LGE intensity, $60 \%$ for $60-64 \%$ LGE intensity and $40 \%$ for a $>64 \%$ normalised LGE intensity [7]. In terms of electrophysiological changes, elevated TGF- 31 was represented by modification of ionic properties. In regions with an LGE intensity $>3$ standard deviations above the mean of the blood pool, maximal ionic conductances were rescaled $(50 \% \mathrm{gK} 1,60 \% \mathrm{gNa}$ and $50 \%$ gCaL) [8] [9].

The effects of repolarization heterogeneity were incorporated as different maximal ionic conductances of the Courtemanche et al human atrial model [8] [10].

\subsubsection{AF simulation and post-processing}

Initial conditions for each simulation corresponded to four spiral wave re-entries [6] [11] and these were used to enable equivalent initiation of AF for each anatomy. For this set-up, the Universal Atrial Coordinate system [3] was used to register an activation time field consisting of two 
Archimedean spirals on each of the posterior and anterior walls, with opposite chirality for adjacent spirals. This is demonstrated in Figure 3.

The data obtained during the AF simulations was postprocessed to identify phase singularity locations for 15 seconds of arrhythmia data. The methodology used for spatial phase singularity (PS) density map calculation has been detailed previously [8].

(A) Define local activation times in 2D UAC

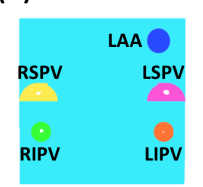

(B) Initial conditions

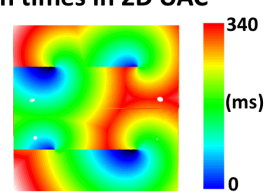

(C) AF isopotential plots
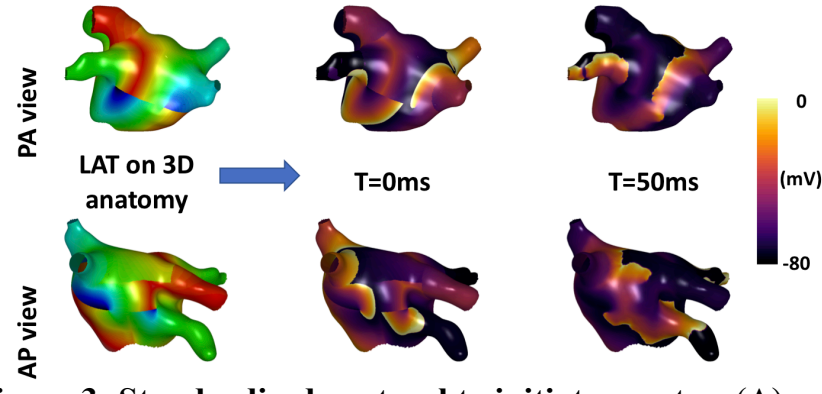

Figure 3: Standardised protocol to initiate reentry. (A) A local activation time (LAT) field was defined analytically as four spiral waves in 2D universal atrial coordinates (UAC). (B) This LAT field was mapped to the atrial mesh (first column) and converted to initial conditions for the voltage distribution and state variables using a pre-pacing functionality in openCARP (second column). (C) AF isopotential snapshots $50 \mathrm{~ms}$ post AF induction. PA: posteroanterior view; AP: anteroposterior view.

\subsubsection{Modelling ablation}

In this study, models were ablated using pulmonary vein isolation (PVI). PVI was modelled as wide-area encirclement of the pulmonary veins and applied at a fixed distance threshold from the left atrial body-pulmonary vein junctions. This ablation approach was strictly defined to ensure consistent lesions across anatomies. Ablation outcome was classified $1.5 \mathrm{~s}$ post-ablation as $\mathrm{AF}$, atrial tachycardia (AT) or termination.

\section{Results}

Each model was generated in approximately 4.5 hours, on a laptop with 4 cores and 15 GB of RAM. Figure 4 shows isopotential plots $1.5 \mathrm{~s}$ post PVI for the models of the cohort. PVI ablation simulations were classified as either AF (non-responders, shown with a white background in Figure 4), AT or termination (responders,

grey background). Table 1 shows the number of cases for each outcome, which were not different between the paroxysmal and persistent AF patient anatomies of the cohort. Overall, PVI ablation was effective in $20 \%$ of cases. The mean total PS hotspot area was $27.7 \pm 9.12 \mathrm{~cm}^{2}$ pre-ablation and the mean hotspot area for which ablation was not attempted was $21.0 \pm 8.02 \mathrm{~cm}^{2}$. The mean area of non-ablated tissue in the post-ablation mesh was $90.1 \pm$ $22.4 \mathrm{~cm}^{2}$.
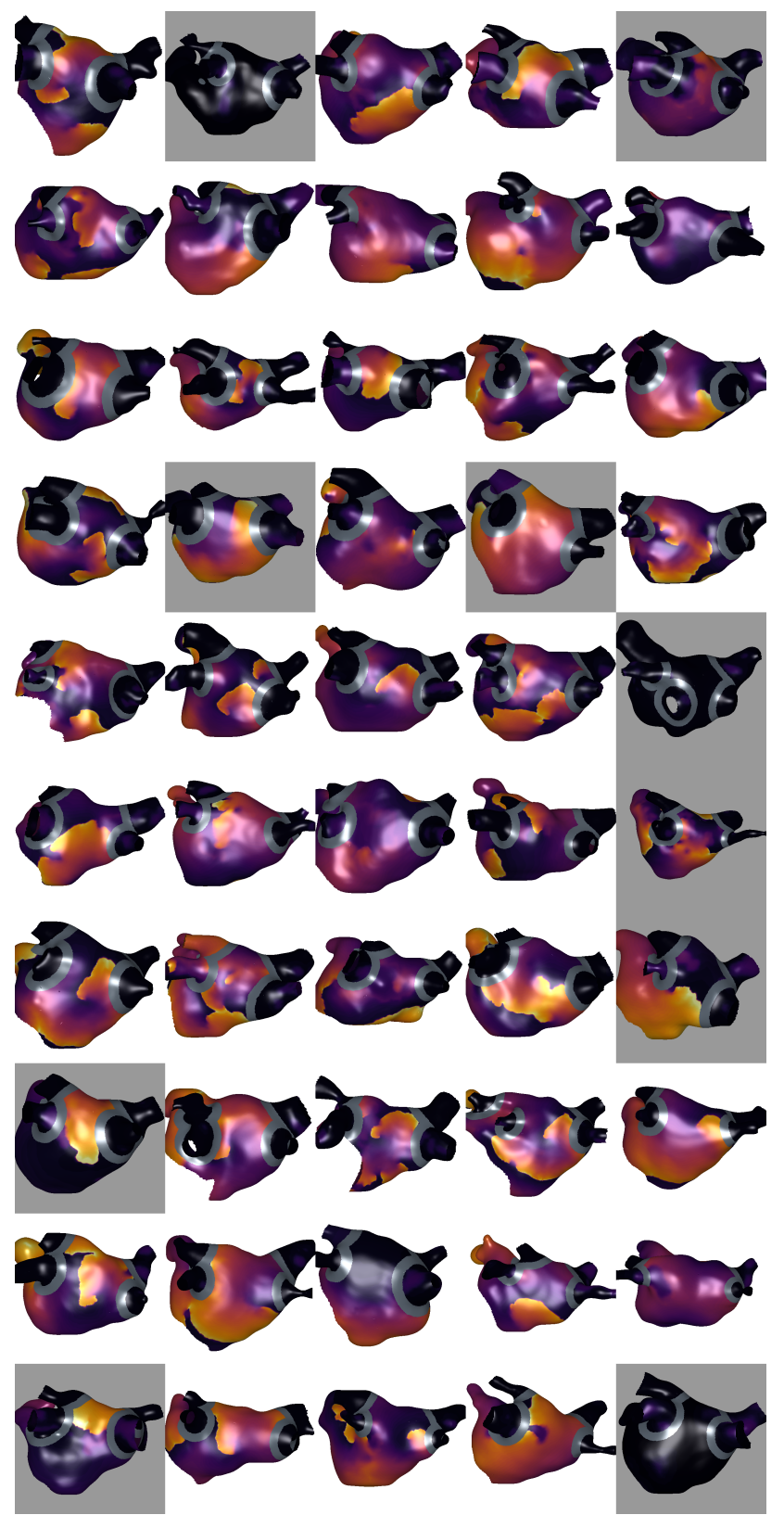

Figure 4: Transmembrane potential plots $1.5 \mathrm{~s}$ post PVI. The first four rows are paroxysmal cases, the last six rows are persistent cases. Acute ablation non-responders (AF continues) are shown with a white background, while responders (AT or termination) have a grey background. 
Table 1. PVI ablation approach simulation outcomes.

\begin{tabular}{lll}
\hline Outcome & Persistent & Paroxysmal \\
& AF & AF \\
\hline AF & 24 & 16 \\
AT & 4 & 2 \\
Termination & 2 & 2 \\
\hline
\end{tabular}

\section{Discussion}

The modelling pipeline developed in this study enables consistent construction of a virtual patient cohort of personalised anatomical models. We demonstrated that LGE-MRI segmentation data can be used to produce anatomical models which after post-processing, fibre incorporation and patient-specific fibrotic remodelling, are suitable for simulation. These models can be produced on a time scale compatible with the clinical setting.

We have demonstrated that the models produced by this pipeline can be used for both $\mathrm{AF}$ and ablation simulations. The post-processing of these simulations allows improved understanding of the effects of patient-specific mechanisms, atrial anatomy, electrical and structural substrate on ablation treatment outcome. Using this pipeline to simulate and predict patient-specific responses to ablation could lead to improved patient care. In this study we simulated only PVI ablation approach. However, this modelling pipeline could also be extended to test other ablation approaches.

We constructed left atrial bilayer models, but other model types may also be appropriate. For example, a volumetric instead of a bilayer model could be used [12]. The use of a volumetric model could lead to more accurate patient-specific ablation treatment response predictions. However, it must also be considered that the simulation time required for a volumetric model is greater than that required for a bilayer model. A volumetric model may therefore be comparatively less appropriate for the clinical setting than the bilayer model, in terms of clinical timescales. Future studies should further consider the most appropriate model type to use within this pipeline for constructing a virtual patient cohort for simulating $\mathrm{AF}$ ablation.

\section{Acknowledgements}

CHR acknowledges a Medical Research Council Skills Development Fellowship (MR/S015086/1). SAN acknowledges support from the UK Engineering and Physical Sciences Research Council (EP/M012492/1, NS/A000049/1 and EP/P01268X/1), the British Heart Foundation (PG/15/91/31812, PG/13/37/30280) and Kings Health Partners London National Institute for Health
Research (NIHR) Biomedical Research Centre. This work was supported by the Wellcome/EPSRC Centre for Medical Engineering [WT 203148/Z/16/Z].

\section{References}

[1] A. Verma et al., "Approaches to catheter ablation for persistent atrial fibrillation.," N. Engl. J. Med., vol. 372, no. 19 , pp. 1812-22, 2015 .

I. Sim et al., "Reproducibility of Atrial Fibrosis Assessment Using CMR Imaging and an Open Source Platform," JACC Cardiovasc. Imaging, vol. 12, no. 10, pp. 2076-2077, 2019.

[3] C. H. Roney et al., "Universal atrial coordinates applied to visualisation, registration and construction of patient specific meshes," Med. Image Anal., vol. 55, pp. 65-75, 2019.

[4] S. Labarthe et al., "A bilayer model of human atria: mathematical background, construction, and assessment.," Europace, vol. 16 Suppl 4, pp. iv21-iv29, Nov. 2014

[5] F. Pashakhanloo et al., "Myofiber architecture of the human atria as revealed by submillimeter diffusion tensor imaging," Circ. Arrhythmia Electrophysiol., vol. 9, no. 4, pp. 1-9, 2016.

[6] C. H. Roney et al., "Constructing a human atrial fibre atlas," Ann. Biomed. Eng., 2020.

[7] M. W. Krueger et al., "Patient-specific modeling of atrial fibrosis increases the accuracy of sinus rhythm simulations and may explain maintenance of atrial fibrillation," J. Electrocardiol., vol. 47, no. 3, pp. 324 328, 2014.

[8] C. H. Roney et al., "Modelling methodology of atrial fibrosis affects rotor dynamics and electrograms," Europace, 2016.

[9] S. Zahid et al., "Patient-derived models link re-entrant driver localization in atrial fibrillation to fibrosis spatial pattern," Cardiovasc. Res., 2016.

[10] M. Courtemanche, R. J. Ramirez, and S. Nattel, "Ionic mechanisms underlying human atrial action potential properties: insights from a mathematical model.," Am. J. Physiol., vol. 275, no. 1 Pt 2, pp. H301-21, Jul. 1998.

[11] E. Matene, A. Vinet, and V. Jacquemet, "Dynamics of atrial arrhythmias modulated by time-dependent acetylcholine concentration: a simulation study.," Europace, vol. 16 Suppl 4, pp. iv11-iv20, Nov. 2014.

[12] C. M. Augustin et al., "The impact of wall thickness and curvature on wall stress in patient-specific electromechanical models of the left atrium," Biomech. Model. Mechanobiol., vol. 19, pp. 1015-1034, 2019.

Address for correspondence:

Dr Caroline Roney

School of Biomedical Engineering \& Imaging Sciences Rayne Institute, $4^{\text {th }}$ Floor, Lambeth Wing St Thomas' Hospital Westminster Bridge Road, London, SE1 7EH

Caroline.roney@kcl.ac.uk 